\title{
Acquisition of concurrent conditional discriminations in rats with ibotenate lesions of hippocampus and of subiculum
}

\author{
LEONARD E. JARRARD \\ Washington and Lee University, Lexington, Virginia \\ and \\ T. L. DAVIDSON \\ Virginia Military Institute, Lexington, Virginia
}

\begin{abstract}
Cells in the hippocampus and subiculum of rats were removed with injections of ibotenic acid, and the animals were subsequently trained on two concurrent Pavlovian conditional discriminations. In both discriminations, a stimulus (i.e., a facilitator) signaled the reinforcement of another stimulus (i.e., a target), but the discriminations differed in that one involved an increased memory load for its solution. Both the animals without a hippocampus and those with extensive damage to the subiculum learned the conditional discriminations at the same rate as did controls. Data obtained from transfer and probe trials indicated that discrimination performance was not due to either direct association of reinforcement with unique configural cues or the formation of direct associations between facilitators and reinforcement. The failure to find that removing the hippocampus affects either original learning (present results) or performance of complex Pavlovian conditional discriminations learned before the structure is removed (Davidson \& Jarrard, 1989) seem to indicate that the hippocampus is not involved in acquisition, retention, or performance of complex conditional discriminations.
\end{abstract}

In a recent experiment designed to study the role of the hippocampus in the performance of Pavlovian conditional discriminations, we found that ibotenate lesions of the hippocampus did not disrupt retention of two complex, concurrent conditional discrimination problems that had been learned before the operations (Davidson \& Jarrard, 1989). The failure to find that hippocampal lesions affect performance of conditional discriminations does not agree with earlier results reported by Ross, Orr, Holland, and Berger (1984). In the Ross et al. experiment, aspiration lesions of the hippocampus produced deficits in both acquisition and retention of a conditional discrimination but had no effect on nonconditional discrimination performance. An important difference between these studies is in the nature and extent of the brain damage. In our experiment, the hippocampus was removed with an axonsparing neurotoxin that permits damage to be limited to the intended area, whereas the aspiration approach used by Ross et al. results in extensive damage to extrahippocampal neural elements.

The present experiment was designed to extend our earlier research by studying the effects of ibotenate lesions of the hippocampus and of the subiculum on the acquisition of conditional discriminations. There is a grow-

This research was supported by National Science Foundation Grants BNS 8507259 and 8809208 to L.E.J. and NIH Grant NS24944-01 to T.L.D. Requests for reprints may be sent to L. E. Jarrard, Department of Psychology, Washington and Lee University, Lexington, VA 24450. ing body of evidence indicating that rats without a hippocampus are able to remember and carry out complex spatial tasks if the tasks are learned before the structure is removed, but are unable to learn the same tasks if the hippocampus is removed before training (Bouffard \& Jarrard, 1988; Jarrard, 1986; Morris, Tweedie, Schenk, \& Jarrard, 1989). These findings seem to indicate that the hippocampus may be necessary for the acquisition of spatial information, but is not the storage site and is not necessary for retrieval of such information. Given these results, and our finding that postoperative performance of conditional discriminations is unaffected if training occurs before the hippocampus is removed, it seems important to see if rats can learn complex conditional discriminations without a hippocampus.

In the present experiment, rats had either the hippocampus or the subiculum removed with multiple injections of small amounts of ibotenic acid. Animals with subiculum lesions were included since this brain area is seen as being the major output pathway from the hippocampal formation (Swanson, 1979). Following recovery from the operations, the animals were trained on two concurrent conditional discriminations that differed in memory load.

The animals then underwent additional testing with transfer and probe trials to see if the groups differed in how the discriminations were learned. The transfer test assessed whether or not the rats solved the conditional discrimination problems by directly associating the facili- 
tator with food reinforcement. This was done by testing the degree to which the facilitators elicited headjerking when they were presented alone. The possibility that the rats were solving the discriminations on the basis of learning about a unique or configural cue arising from each facilitator and target compound (e.g., Rescorla, 1972) was also examined by substituting different transfer targets for the originally trained targets. If the facilitators also promoted responding to the novel transfer targets, this would argue against the configural learning hypothesis. In addition, the transfer test examined the hypothesis that the facilitators had become conditioned modulators of the strength of the associative relationship between their targets and reinforcement (Rescorla, 1985). If this were the case, a given facilitator would not be expected to promote responding to a transfer target that had no history of reinforcement, and therefore no associative connection with reinforcement.

\section{METHOD}

\section{Subjects and Apparatus}

The subjects were 32 naive male Sprague-Dawley rats that weighed between 250 and $300 \mathrm{~g}$ at the beginning of the experiment. The daily food ration was adjusted so that body weights were reduced to $85 \%$ of their ad lib weights. A $1.5 \%$ gain in body weight was allowed each week for the duration of the experiment. The animals were housed individually and the light period of their 12:12-h light:dark cycle began at $0600 \mathrm{~h}$. One control animal failed to learn the tasks, so the data for this animal were discarded.

The apparatus was the same as that described in detail by Davidson and Jarrard (1989). Briefly, there were four identical, standard conditioning chambers with front and back walls of clear acrylic plastic. A pellet dish was located on a side wall, and a 6-W bulb over the dish provided constant, low-level chamber illumination. The chambers were enclosed in a sound-resistant shell with a clear Plexiglas front that permitted behavioral observations. A 25-W bulb located above the center of each chamber provided a 3-Hz flashinglight stimulus. A tubular $40-\mathrm{W}$ bulb, $14 \mathrm{~cm}$ in length, was located slightly below one Plexiglas wall of each chamber and served as a second light stimulus. Auditory stimuli consisting of white noise, a $1500-\mathrm{Hz}$ tone, and a $2500-\mathrm{Hz}$ tone were provided via a speaker mounted on the interior shell wall. A $10-\mathrm{Hz}$ clicker was mounted on the center of the back wall of the shell.

A video camera located $2.09 \mathrm{~m}$ in front of the chambers was positioned so that activity in all four chambers could be recorded simultaneously. The videotaped behavior of the rats was played back for scoring.

\section{Procedure}

The animals were assigned to one of four groups (hippocampal, subiculum, operated control, and unoperated control) of 8 rats each. The surgical procedures used to remove the hippocampus and the subiculum are described elsewhere (Jarrard, 1989). Following a 20-day postoperative recovery period, each rat was given one session of magazine training, which consisted of automatic delivery of 10 food pellets $(45 \mathrm{mg}, \mathrm{P}$. J. Noyes Co.) on a variable-time 1 -min schedule. Pavlovian conditioning on two concurrent conditional discrimination problems began 2 days after magazine training. One problem was of the form F1T1 +, T1 - On F1T1 + trials, a diffuse visual stimulus (F1) was presented for $15 \mathrm{sec}$. An auditory tone stimulus (T1) overlapped the final $5 \mathrm{sec}$ of the light, with reinforcement immediately following cotermination of both stimuli.
On $\mathrm{T} 1$ - trials, the 5 -sec tone was presented by itself without reinforcement. The other problem was of the form $\mathrm{F} 2-\mathrm{T} 2+, \mathrm{T} 2-$. On F2 _- T2 + trials, a 5-sec presentation of a different visual stimulus (F2) was followed by a 5-sec trace interval, which in turn was followed by a 5 -sec presentation of a different auditory stimulus (T2) and then termination of $T 2$ with reinforcement. Trials consisting of presentation of $\mathrm{T} 2$ by itself were not followed by reinforcement. For half of the subjects, a flashing overhead light served as F1, and a steady light served as F2. The F stimuli (flashing or steady light) were reversed for the remaining rats. The auditory target stimuli were also counterbalanced.

Each animal received three presentations of each of the four trial types, for a total of 12 trials during each session. The intertrial interval (ITI) varied around a mean of $2.5 \mathrm{~min}$. The trials were presented in two different quasirandom sequences, which were alternated every other day. The animals received one session per day for 32 days.

On the day following conditional discrimination training, the animals were given 2 days of training in which the presentation of white noise (TR) for $5 \mathrm{sec}$ terminated with food reinforcement. This was followed by 3 days of extinction in which the 5-sec TR was not followed by food. The rats received 12 presentations of TR on each day of training and extinction, with an average ITI of $2.5 \mathrm{~min}$. Two days of transfer testing began 4 days after extinction. Each transfer session consisted of two presentations each of eight different trial types. There were 15 -sec presentations each of $\mathrm{F} 1$ - and F2 - , and 5-sec presentations of TR - and a novel tone, $\mathrm{N}-$, that had never been experienced. $\mathrm{F} 1$ - was presented for $15 \mathrm{sec}$ with TR - overlapping the last $5 \sec ($ F1TR - ), and there were trials with $\mathrm{F} 1$ - presentations and $\mathrm{N}-$ overlapping the last $5 \sec (\mathrm{F} 1 \mathrm{~N}-)$. The rats also received two trials of 5-sec F2 with TR being presented after a 5-sec interval (F2_TR-), and then trials with 5 sec of F2, the 5-sec gap, and then $5 \mathrm{sec}$ of $\mathrm{N}-\left(\mathrm{F} 2 \_\mathrm{N}\right)$. The stimuli were counterbalanced across groups, and the mean ITI was $2.5 \mathrm{~min}$.

\section{Scoring}

Learning was assessed using the videotaped recordings and a behavioral observation technique similar to that described by Davidson and Rescorla (1986). Briefly, the six categories of behavior recorded were headjerking, rearing, standing motionless in front of the food magazine, locomotion, magazine-oriented activity (other than headjerking), and other. Three observations of each rat were made during the $15-\mathrm{sec}$ period of each trial. This trial period began with the onset of the light (F1 or F2) on F1T1+ and F2 _ T + trials, and began $10 \mathrm{sec}$ before the onset of $\mathrm{T} 1-$ and $\mathrm{T} 2-$ when these cues were presented alone. Paced by an auditory signal presented every $1.25 \mathrm{sec}$, the observer shifted his gaze from chamber to chamber so that he observed each rat once every $5 \mathrm{sec}$. Only one behavior was recorded on each observation.

\section{RESULTS AND DISCUSSION}

\section{Anatomical}

The nature and extent of the damage to the hippocampus and the subiculum resulting from injections of ibotenic acid was similar to that described in several recent papers (Davidson \& Jarrard, 1989; Jarrard, 1986, 1989). The loss of cells in the hippocampus was extensive at all dorsal-ventral levels, and included both pyramidal cells (CA1-CA4) as well as the granule cells in the dentate gyrus. Although there was some minimal sparing of CA1 cells and dentate gyrus in several animals in the hippocampal group, the surviving cells were usually unilateral and few in number. Damage to extrahippocampal areas was 
also minimal. Of special concern was damage to the subiculum, and although there was some additional loss of cells in several animals at a mid-dorsal-ventral level, this damage was small in amount.

Examination of stained brain sections of animals from the subiculum group indicated that there was extensive damage to the subicular area. The resulting damage was more selective and complete in the middle third of the area, with some bilateral sparing of subiculum cells at the most dorsal, and especially at the ventral areas. At several levels there was some loss of granule cells in the dentate gyrus and CA1 cells adjacent to the subiculum. In addition, there was obvious atrophy of the area of the dentate gyrus in most rats. In the animals in the operated control group, there was no obvious brain damage other than an occasional increase in glial cells, indicating penetration of the pipette through the cortex.

\section{Behavioral}

Conditional discrimination training. Consistent with other studies (e.g., Davidson \& Jarrard, 1989), headjerking emerged as the primary index of learning. Figure 1 shows the mean percentage of headjerking observed during the target period (i.e., the last $5 \mathrm{sec}$ ) of each reinforced $(+)$ and nonreinforced $(-)$ trial type, during six 2-day blocks of training. Data from the control, hippocampal, and subiculum groups are presented, respectively, in the left, center, and right panels of Figure 1.

Although the rats solved both discrimination problems (as indicated by the fact that each group headjerked more on $\mathrm{F} 1 \mathrm{~T} 1+$ and $\mathrm{F} 2$ _ $\mathrm{T} 2+$ trials than on $\mathrm{T} 1-$ or $\mathrm{T} 2-$ trials), there was no evidence of group differences in discrimination performance (see Figure 1). Analysis of variance utilizing blocks, sessions (within blocks), trial type (compound vs. target alone), and problem type (trace vs. no trace) as within-subjects factors and groups as a between-subjects factor was used to evaluate acquisition performance. This analysis revealed a significant difference due to trial type $[F(1,28)=629.77, p<.01]$, confirming that more headjerking occurred on F1T1 + and $\mathrm{F} 2$ - $\mathrm{T} 2+$ compound trials than on $\mathrm{T} 1-$ and $\mathrm{T} 2$ - targetalone trials. A significant trial type $\times$ blocks interaction was also obtained $[F(5,140)=55.16, p<.01]$, substantiating that the effect of discrimination performance improved over sessions. Although differences in headjerking between compound trials and target-alone trials appeared to be smaller for the trace problem than for the no-trace problem, the two-way interaction between trial type and problem type failed to reach statistical significance $[F(1,28)=3.76, p>.06]$. However, there

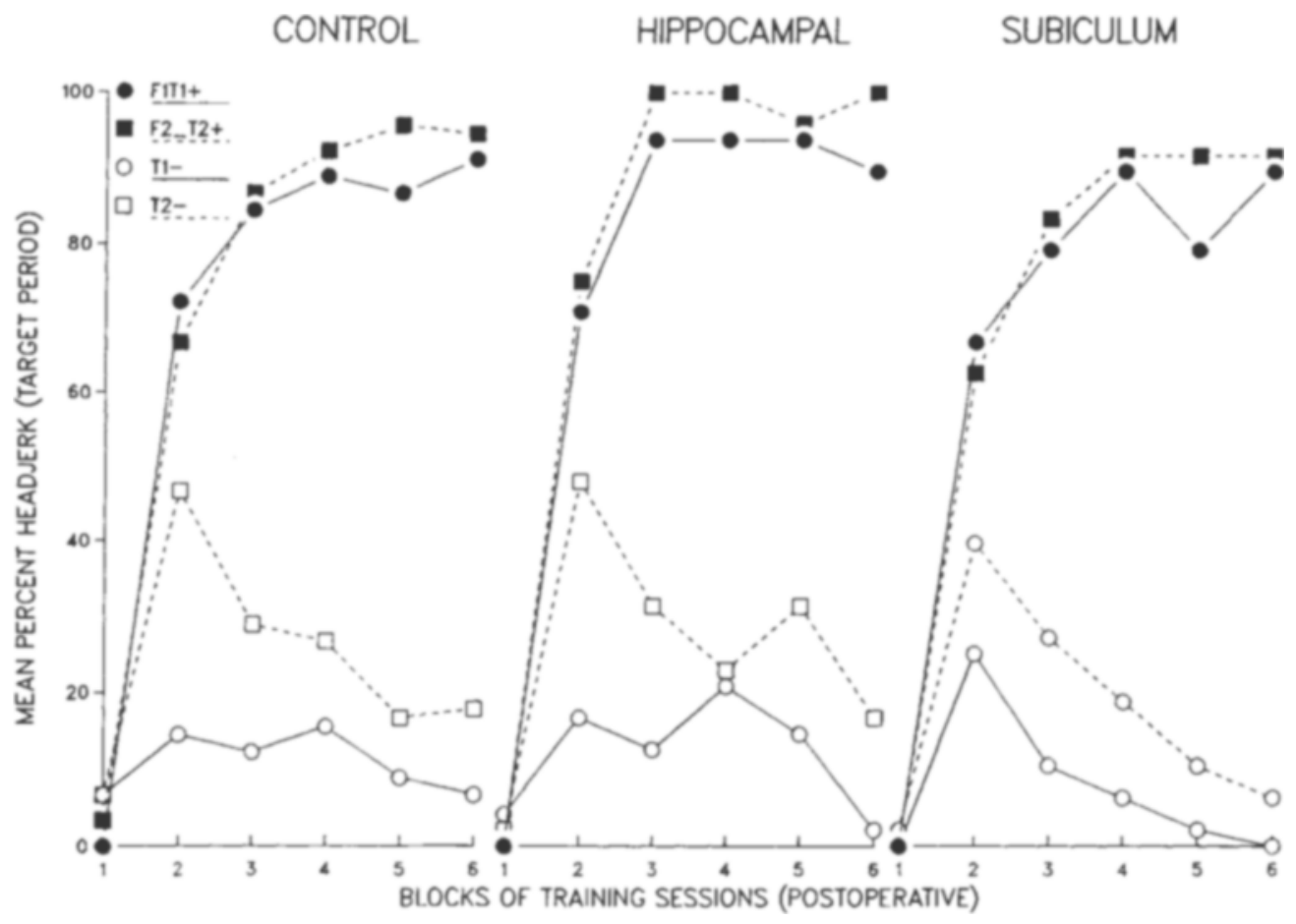

Figure 1. Mean percentage of headjerking during acquisition of the no-trace and trace problems in the control (left), hippocampal (middle), and subiculum (right) groups. Responding is shown separately for reinforced (F1T1 $+, F 2, T 2+)$ and nonreinforced $(\mathrm{T} 1-, \mathrm{T} 2-)$ trials. Days 1 and 2 comprised the first block of training, whereas Days 5 and 8,11 and 14, 17 and 20, 23 and 26, and 29 and 32 comprised Blocks 2-6, respectively. Performance is shown separately for each of the trial types that constituted the two discrimination problems. Trial types designated with a 1 comprised the no-trace problem, whereas trial types designated with a 2 comprised the trace problem. 
was no reliable main effect due to groups, there were no reliable group $\times$ trial type or group $\times$ problem type interactions, nor was there a reliable group $x$ trial type $x$ problem type interaction [largest $F(2,28)=1.11$, $p>.34$, for the group $\times$ problem type interaction]. Hence, the effects of trial type and problem type did not differ among the groups.

Transfer testing. Despite the lack of differences in headjerking among the control, hippocampal, and subiculum groups, it was nonetheless possible that the groups solved the conditional discrimination problems in different ways. The transfer test attempted to detect such differences.

As preparation for the transfer test, all rats were given 2 days of reinforced acquisition training to a novel target, followed by 3 days of extinction. The mean percentage of acquisition trials on which headjerking was observed was $72.9,64.1$, and 75.8 for the hippocampal, subiculum, and control groups, respectively. Mean headjerking on the last day of extinction was $38.5 \%$ for hippocampals, $28.1 \%$ for subiculum animals, and $38.9 \%$ for controls. The differences among groups failed to achieve statistical significance during either acquisition or extinction [largest $F(2,28)<1]$.

Table 1 shows the mean percentage of target-period headjerking during the transfer test for each type of test trial. All three groups showed the same general pattern of responding on each type of transfer trial. As can be seen in Table 1, both F1 and F2 promoted responding to the previously trained and extinguished transfer target TR. Reliably more headjerking occurred on respective F1TR $[F(1,28)=50.47, p<.01]$ and $\mathrm{F} 2$ _TR $[F(1,28)=$ $44.62, p<.01$ ] compound trials than on trials in which TR was presented alone. These differences in headjerking did not vary by group [largest $F(2,28)=0.784$, $p>.47$, for the group $\times \mathrm{F} 2$ _ TR vs. TR interaction]. Hence, for all groups, both $F 1$ and $F 2$ facilitated responding to a stimulus different from that with which it was originally trained. This finding reduces the plausibility of the notion that the conditional discrimination performance was based on learning about configural or unique cues. Furthermore, more headjerking was elicited on F1TR compound trials than on trials in which F1 was presented alone $[F(1,28)=37.90, p<.01]$. Similarly, the F2_TR compound elicited more headjerking than did F2 alone $[F(1,28)=32.07, p<.01]$. Neither of these differences varied by group [largest $F(2,28)=1.254$, $p<.29$, for the group $\times$ F2_TR vs. F2 interaction].
These outcomes indicate that the capacity of F1 and F2 to promote headjerking was not solely due to their direct associations with reinforcement for any of the groups.

It was also found that the capacity of both F1 and F2 to augment headjerking to a target depended on the training history of the target. Both F1 and F2 promoted more headjerking to the previously trained and extinguished transfer target TR than to the untrained novel transfer target $\mathrm{N}$. Differences between F1TR and F1N trials and between $\mathrm{F} 2$ _ TR and $\mathrm{F} 2 \mathrm{~N}$ trials were significant $[F(2,28)=$ $22.6, p<.01$, and $F(2,28)=53.82, p<.01$, respectively], and did not vary by group [largest $F(2,28)<1$, $p>.40$ ]. This finding is consistent with the hypothesis that facilitators promote behavior indirectly by lowering the threshold for activation of associations between other stimuli and reinforcement (Rescorla, 1985). This modulatory capacity was apparently undisturbed by destruction of the hippocampus or subiculum.

In the theory of hippocampal functioning recently proposed by Sutherland and Rudy (1989), the hippocampal formation is seen as being the neural basis for the acquisition and storage of configural associations among events. Sutherland and Rudy interpret Ross et al.'s (1984) results as providing support for the configural theory, since in performing the serial-compound discrimination, "the animal discriminates between the two kinds of tone presentations by forming a configural representation of the light-tone serial compound that differs from the configural representation of the tone alone'" (p. 136). The authors do point out that differential responding in the serial-compound discrimination problem could be due to summation of excitatory properties arising from the association of the light and tone with reinforcement. In this case, the excitatory properties of the light and tone presented together would exceed that of the tone alone. If performance of conditional discriminations is due to summation, Sutherland and Rudy predict that damage to the hippocampus would not impair performance.

Since the rules for summation among different stimuli or among responses with different topographies are unknown, it is not possible to specify in advance when conditional discrimination performance is likely to be due to summation and when it is likely to be due to the formation of unique configural representations. At least one aspect of our transfer results reduces the plausibility of a summation account of the present findings. Both the hippocampal and the subiculum groups headjerked more to $F 2$ than to F1 when each was presented alone. None-

Table 1

Mean Percentage of Headjerking (Target Period) for Each Group on Each Type of Transfer-Test Trial

\begin{tabular}{lcccccccc}
\hline & \multicolumn{7}{c}{ Transfer-Test Trial } \\
\cline { 2 - 9 } \multicolumn{1}{c}{ Group } & F1TR & TR & F2_TR & F1N & N & F2__N & F1 & F2 \\
\hline Hippocampal & $\mathbf{8 7 . 5}$ & 25.0 & 87.5 & 56.3 & 12.5 & 25.0 & 43.8 & 65.6 \\
Subiculum & 75.0 & 31.3 & 68.8 & 25.0 & 6.3 & 31.3 & 25.0 & 34.4 \\
Control & 66.7 & 23.3 & 83.3 & 40.0 & 3.3 & 23.3 & 40.0 & 41.7 \\
\hline
\end{tabular}


theless, these groups headjerked either the same (hippocampal) or more (subiculum) to F1 than to F2 when each was combined with TR. Controls showed comparable levels of headjerking to both F1 and F2 when they were presented alone, yet they also tended to headjerk more on F2_.TR than on F1TR compound trials. It would seem very difficult to specify summation rules that could account for this pattern of transfer results. Although there are design and procedural differences between our study and that of Ross et al. (1984), there is little reason to think that these differences would support summation more in one study than in the other.

The present results have implications for other recent theories concerning hippocampal function. Both the "working-memory" (Olton, Becker, \& Handelmann, 1979 ) and the "temporary memory buffer" (Rawlins, 1985 ) theories hypothesize a role for the hippocampus in the processing of certain types of memory. There is little support in either our present or our previous study (Davidson \& Jarrard, 1989) for such mnemonic interpretations of hippocampal function, since animals without a hippocampus were able to learn and perform even the conditional discrimination with the increased memory load (F2_T2) as well as controls could. Our results are more in agreement with the cognitive mapping theory of O'Keefe and Nadel (1978), since they predict that in nonspatial tasks no deficit should result from removing the hippocampus. It is important to point out that even though removing the hippocampus had no effect on either acquisition or retention of the complex conditional discriminations that we employed, animals with similar lesions are impaired in learning spatial tasks (see introduction). Thus, ibotenate hippocampal lesion does result in a behavioral deficit if the appropriate task is chosen.

Although acquisition of the conditional discriminations was not affected by damage to either the hippocampus or the subiculum, we have found that combined damage involving both structures does affect performance in other tasks. Specifically, when ibotenate lesions of the hippocampus also included extensive loss of cells in the subiculum, rats were found to be as imparied as those with aspiration hippocampal lesions in performing a preoperatively learned spatial task (Jarrard, 1986). It is not known whether conditional discriminations would be affected by combined ibotenate damage to the hippocampus and subiculum, but aspiration lesions do impair performance of such tasks (Ross et al., 1984). Thus, it may be the case that extensive damage to several hippocampalformation structures is needed in order to obtain impairments in conditional discrimination performance.

The failure to find that removing the hippocampus affects either acquisition (present results) or retention (Davidson \& Jarrard, 1989) of complex Pavlovian conditional discriminations is in direct conflict with the results reported by Ross et al. (1984). There are obvious procedural differences between the studies. Ross et al. trained their rats concurrently on one conditional and one nonconditional discrimination task, whereas we trained our rats concurrently on two different conditional discriminations. Even small procedural differences can be important, as is evident from the research of Loechner and Weisz (1987). Those investigators found that when lights and tones were counterbalanced in the featurepositive discrimination paradigm, the stimulus that was used as the conditional cue determined whether a hippocampal-lesion deficit was found. However, in both our studies and those of Ross et al., similar stimuli were used for both the facilitators (lights) and the targets (tone, clicker, white noise).

An important difference between our research and that reported by Ross et al. (1984) has to do with the extent of the underlying brain damage. In our studies, the hippocampus was removed using multiple injections of small amounts of ibotenic acid, a lesioning technique that minimizes damage to adjacent structures and fibers of passage (Jarrard, 1989). The aspiration approach employed by Ross et al. is known to interrupt many extrahippocampal axons that pass through the alveus and fimbria, to involve direct damage to subiculum, presubiculum, cingulate cortex, and cingulum, and to cause damage to the vasculature. Thus, the more selective removal of the cells in the hippocampus that is possible with ibotenic acid should provide a better answer to the question of whether or not the hippocampus is the necessary neural substrate for the acquisition and performance of conditional discriminations.

In our previous study, rats learned the two concurrent conditional discriminations before the hippocampus was removed, and retention testing revealed that performance was unaffected (Davidson \& Jarrard, 1989). These findings suggest that the storage of the relevant information is not in the hippocampus and that this structure is not necessary for the retrieval and handling of complex conditional discrimination information. The present results extend these findings by showing that without a hippocampus, and with damage to the subiculum, rats learn the conditional discriminations in a similar way as controls do, indicating that the hippocampus is not involved in the acquisition of these tasks. Thus, unlike the important and necessary role the structure seems to play in the acquisition (but not retention or retrieval) of complex spatial information (see introduction), our results suggest that the hippocampus may not be involved in acquisition, retention, or performance of complex Pavlovian conditional operations.

\section{REFERENCES}

Bouffard, J.-P., \& JARRARD, L. E. (1988). Acquisition of a complex place task in rats with selective ibotenate lesions of hippocampal formation: Combined lesions of subiculum and entorhinal cortex vs. hippocampus. Behavioral Neuroscience, 102, 828-834.

Davidson, T. L., \& JARRARD, L. E. (1989). Retention of concurrent conditional discriminations in rats with ibotenate lesions of hippocampus. Psychobiology, 17, 49-60.

Davidson, T. L., \& Rescorla, R. A. (1986). Transfer of facilitation in the rat. Animal Learning \& Behavior, 14, 380-386.

JARRARD, L. E. (1986). Selective hippocampal lesions and behavior: 
Implications for current research and theorizing. In R. L. Isaacson \& K. H. Pribram (Eds.), The hippocampus (Vol. 4, pp. 93-126). New York: Plenum.

JARRARD, L. E. (1989). On the use of ibotenic acid to selectively lesion different components of the hippocampal formation. Journal of Neuroscience Methods, 29, 251-259.

LOECHNER, K. J., \& WEISZ, K. J. (1987). Hippocampectomy and featurepositive discrimination. Behavioral Brain Research, 26, 63-73.

Morris, R. G. M., Twefdie, F., SChenk, F., Jarrard, L. E. (1989). Dissociation between components of spatial memory after ibotenate lesions of the hippocampus. Sociery for Neuroscience Abstracts, 15 , 609.

O'KeEFE, J., \& NADEL, L. (1978). The hippocampus as a cognitive map. London: Oxford University Press.

Olton, D., Becker, J. T., \& Handelmann, G. E. (1979). Hippocampus, space, and memory. Behavioral \& Brain Sciences, 2, 313-365.

Rawlins, J. N. P. (1985). Associations across time: The hippocampus as a temporary memory store. Behavioral \& Brain Sciences, 8 , 479-496.
Rescorla, R. A. (1972). "Configural" conditioning in discrete-trial bar pressing. Journal of Comparative \& Physiological Psychology, 79, 307-317.

Rescorla, R. A. (1985). Facilitation and inhibition. In R. R. Miller \& N. E. Spear (Eds.), Information processing in animals: Conditioned inhibition (pp. 299-326). Hillsdale, NJ: Erlbaum.

Ross, R. T., Orr, W. B., Holland, P. C., Berger, T. W. (1984). Hippocampectomy disrupts acquisition and retention of learned conditional responding. Behavioral Neuroscience, 2, 211-225.

SutherLand, R. J., RUDY, J. W. (1989). Configural association theory: The role of the hippocampal formation in learning, memory, and amnesia. Psychobiology, 17, 129-144.

Swanson, L. W. (1979). The hippocampus: New anatomical insights. Trends in Neuroscience, 2, 9-12.

(Manuscript received August 7, 1989; revision accepted for publication November 3,1989 .) 